Case Report

\title{
Multifactorial Origin of Exertional Rhabdomyolysis, Recurrent Hematuria, and Episodic Pain in a Service Member with Sickle Cell Trait
}

\author{
Nyamkhishig Sambuughin (iD, ${ }^{1}$ Mingqiang Ren, ${ }^{1}$ John F. Capacchione, ${ }^{2}$ \\ Ognoon Mungunsukh, ${ }^{3}$ Kevin Chuang, ${ }^{1}$ Iren Horkayne-Szakaly, ${ }^{4}$ \\ Francis G. O'Connor, ${ }^{1}$ and Patricia A. Deuster $\mathbb{D D}^{1}$ \\ ${ }^{1}$ Consortium for Health and Military Performance, Department of Military and Emergency Medicine, \\ Hébert School of Medicine, Uniformed Services University, 4301 Jones Bridge Rd., Bethesda, MD 20184, USA \\ ${ }^{2}$ Department of Anesthesiology, University of Minnesota, 420 Delaware St SE, Minneapolis, MN 55455, USA \\ ${ }^{3}$ Department of Anesthesiology, Hébert School of Medicine, Uniformed Services University, 4301 Jones Bridge Rd., Bethesda, \\ MD 20814, USA \\ ${ }^{4}$ Joint Pathology Center, Defense Health Agency, 606 Stephen Sitter Ave, Silver Spring, MD 20910, USA
}

Correspondence should be addressed to Nyamkhishig Sambuughin; nyamkhishig.sambuughin.ctr@usuhs.edu

Received 15 August 2018; Accepted 24 October 2018; Published 7 November 2018

Academic Editor: Christos Yapijakis

Copyright (C) 2018 Nyamkhishig Sambuughin et al. This is an open access article distributed under the Creative Commons Attribution License, which permits unrestricted use, distribution, and reproduction in any medium, provided the original work is properly cited.

\begin{abstract}
Individuals with Sickle Cell Trait (SCT), generally considered a benign carrier state of hemoglobin S (HbAS), are thought to be at risk for exertional rhabdomyolysis and hematuria, conditions that can also be caused by various other acquired and inherited factors. We report an SCT positive service member with an exertional rhabdomyolysis event, recurrent hematuria with transient proteinuria, and episodic burning pain in the lower extremities. Clinical and genetic studies revealed the multifactorial nature of his complex phenotype. The service member was taking prescription medications known to be associated with exertional rhabdomyolysis. He carried a pathogenic mutation, NPHS2 p.V260E, reported in nephropathy and a new variant p.R838Q in SCN11A, a gene involved in familial episodic pain syndrome. Results suggest that drug-to-drug interactions coupled with the stress of exercise, coinheritance of HbAS and NPHS2 p.V260E, and p. R838Q in SCN11A contributed to exertional rhabdomyolysis, recurrent hematuria with proteinuria, and episodic pain, respectively. This case underscores the importance of comprehensive clinical and genetic evaluations to identify underlying causes of health complications reported in SCT individuals.
\end{abstract}

\section{Introduction}

Sickle Cell Trait (SCT) is a carrier state (HbAS) of a hemoglobin mutation that causes Sickle Cell Disease, one of the most common monogenic inherited blood disorders in humans. It is estimated that about 300 million individuals worldwide have SCT [1]. In the United States, 7.3\% of African Americans, $0.7 \%$ of Hispanics, and $0.3 \%$ Caucasians are carriers of HbAS [2].

Although SCT is generally considered a benign carrier state, it has been associated with a number of health complications such as exertional rhabdomyolysis and hematuria
$[1,3,4]$. Exertional rhabdomyolysis is a metabolic event characterized by the release of muscle contents into the circulation due to exercise related skeletal muscle breakdown. Typically, exertional rhabdomyolysis manifests as muscle pain and weakness, with transient elevation ( $>5-10 \mathrm{x}$ normal) in serum creatine kinase $(\mathrm{CK})$, with or without myoglobinuria [5]. The development of exertional rhabdomyolysis depends on numerous risk factors that can be broadly divided into acquired and inherited. Acquired causes include injuries, medications, supplements, infections, and overexertion [5, 6]. Inherited etiologies include genetic abnormalities in the 
glycolytic and the fatty acid oxidation pathways, and in the mitochondrial respiratory chain [5].

Hematuria in SCT individuals is typically painless and presents as microscopic or gross bleeding. Hematuria thought to occur due to vasoocclusion events that can lead to microinfarctions and renal papillary necrosis $[1,3]$. As with exertional rhabdomyolysis, hematuria can rise from metabolic and/or environmental factors such as dehydration, strenuous exercise, and acidosis. Once these precipitating factors are eliminated, there is usually no recurrence; however, if hematuria persists and its etiology is uncertain, other factors should be considered. A number of studies have demonstrated the effect of genetic variants in renal phenotypes in SCT individuals $[7,8]$.

Pain in SCT individuals has been reported in association with splenic infarction and pulmonary embolism $[1,3]$. In these settings, SCT carriers experience acute pain in chest or left upper abdomen which is again thought secondary to HbAS driven vasoocclusive event. Complications associated with splenic infarction and pulmonary embolism are relatively rare and seen mostly under hypoxic conditions of high altitude $[9,10]$.

Episodic pain reflecting a neurological disorder is clinically distinct from pain associated with HbAS. A growing body of evidence demonstrates a monogenic origin, specifically, the involvement of channelopathies in pain [11]. Recently, autosomal dominant mutations in SCN11A, which encodes Nav1.9, a voltage-gated sodium channel subunit alpha type 9 , have been found in pain syndromes, including episodic familial pain syndrome [11-13].

We report clinical and genetic studies of a service member with SCT who presented with exertional rhabdomyolysis, recurrent hematuria with transient proteinuria, and episodic burning pain in the lower extremities. Study results revealed the multifactorial nature of his complex clinical symptoms with involvement of both acquired and inherited causes. Patient was reported taking prescription medications known to be associated with exertional rhabdomyolysis. Whole exome sequencing (WES) identified a pathogenic mutation in NPHS2 associated with nephropathy [14] and likely pathogenic variant in SCN11A. Our study results underscore the importance of detailed clinical and genetic evaluations to identify underlying causes of health complications reported in SCT individuals.

\section{Case Description}

A 33-year-old African American male service member with SCT presented with an episode of myalgia, muscle stiffness, and a peak CK of 18,867U/L after exercise. His clinical history was significant for chronic exercise associated hematuria, transient proteinuria with creatinine elevation, and severe episodic pain in his lower extremities. He stated that his symptoms had begun four years earlier when he developed shortness of breath and muscle pain and was unable to complete a two-mile run. He was evaluated and found to have hematuria. Since then, he has had multiple episodes of hematuria after exertion with severe muscle pain and stiffness lasting 3-4 days. He reported hydrating well during or following exercise but had also noticed that his calves had become smaller over the years, despite exercise. He had undergone an extensive work-up for the hematuria, to include cystogram and renal ultrasound, but without any definitive diagnosis. He denied a family history of muscle problems and/or adverse reactions to anesthesia; however, a family history of pain or hematuria is unknown. Underlying inflammatory myopathy was ruled out, but the service member was placed on restricted physical activity. However, even with his relative inactivity, he complained of burning pain in his calves and shoulder stiffness two to three times a week. In addition, his health record indicated history of hypertension and depression. Active prescription medications included amlodipine (10-40mg), simvastatin (20mg), sertraline (100mg), and indomethacin (25mg).

Muscle histology showed minimal nonspecific changes (Supplemental Material, Fig. S1). Muscle enzymes were within reference ranges. Electromyography was negative for myopathy. Details regarding nerve conduction studies were not available from clinical history.

2.1. Genetic Study. WES, variants identification and analysis were performed as described previously [15]. Briefly, variants were filtered for minor allele frequency of $<0.1-0.01 \%$ in the general population. Nonsynonymous, splice, stop gain, and stop loss variants were prioritized. A number of in silico algorithms including SWIFT, PolyPhen, and Mutation Tester were utilized to predict the effect of identified variants. The pathogenicity of the variants was assessed per American College of Medical Genetics (ACMG) guidelines [16]. Paralogue annotation method [17] was used to further assess pathogenic effect of variants. The structure of Nav1.9 was predicted and visualized as described by Omasits U. et al. [18]. Sanger sequencing was used to confirm variants identified by WES.

\section{Results}

Prior to WES analysis, common pathogenic variants in $P Y G M, C P T 2, A M P D 1$, and RYR1, genes frequently associated with ER, were analyzed with negative results. WES results for other genes associated with metabolic myopathies implicated in rhabdomyolysis were negative. WES identified seven variants that fulfilled stringent analysis criteria (Table 1). Of deleterious variants identified in this study, p. V260E variant in NPHS2 was previously reported as a pathogenic mutation (Fig. S2) [14]. Remaining variants were analyzed in detail (Supplementary material). Based on information regarding encoded protein function, disease association, disease inheritance pattern, the degree of pathogenicity predicted by various in silico methods, and paralogue analysis, p. R838Q in SCN11A was selected as a candidate pathogenic variant contributing to patient's phenotype. The p. R838Q in SCN11A was predicted to be damaging by various analysis tools used in this study. Protein alignment and paralogue annotation analyses showed that arginine residue at 838 position of SCN11A is highly conserved among various species and also between different subunits of voltage-gated sodium channels (Fig. S3). Importantly, paralogous mutations in 
TABLE 1: Exome sequencing results. Findings are presented by the degree of pathogenicity predicted by various methods.

\begin{tabular}{|c|c|c|c|c|c|c|c|}
\hline \multirow{2}{*}{ Gene } & \multirow{2}{*}{ Variant } & \multirow{2}{*}{ Variant ID $^{\mathrm{a}}$} & \multirow{2}{*}{$\begin{array}{c}\text { ExAC } \\
\text { freq. }(\%)\end{array}$} & \multicolumn{2}{|c|}{ Variant impact prediction ${ }^{\mathrm{b}}$} & \multirow{2}{*}{ ACMG class } & \multirow{2}{*}{$\begin{array}{l}\text { Associated disease } \\
\text { Inheritance Pattern }^{c}\end{array}$} \\
\hline & & & & $\begin{array}{c}\text { Sift } \\
\text { PolyPhen } \\
\end{array}$ & $\begin{array}{c}\text { Mutation } \\
\text { Tester }\end{array}$ & & \\
\hline NPHS2 & $\mathrm{V} 260 \mathrm{E}$ & rs775006954 & 0.005 & Del. & $\begin{array}{l}\text { GScore: } 121 \\
\text { Pp: } 1.0\end{array}$ & Pathogenic & $\begin{array}{c}\text { Steroid Resistant } \\
\text { Nephrotic Syndrome, } \\
\text { AR }\end{array}$ \\
\hline TTN & T587Hfr11Ter & NA & NR & LoF & $\begin{array}{l}\text { GScore: NA } \\
\text { Pp: } 1.0\end{array}$ & VUS & $\begin{array}{c}\text { Various cardiac and } \\
\text { muscle disorders, } \\
\mathrm{AD} / \mathrm{AR} \\
\end{array}$ \\
\hline SCN1A & R604C & rs148371904 & 0.002 & Del. & $\begin{array}{l}\text { GScore: } 180 \\
\text { Pp: } 1.0\end{array}$ & VUS & $\begin{array}{l}\text { Epilepsy, Familial } \\
\text { Migraine, AD }\end{array}$ \\
\hline SCN11A & R838Q & rs149681198 & 0.007 & Del. & $\begin{array}{l}\text { GScore: } 43 \\
\text { Pp: } 0.97\end{array}$ & $\begin{array}{c}\text { Likely } \\
\text { pathogenic }\end{array}$ & $\begin{array}{c}\text { Familial Episodic } \\
\text { Pain, AD } \\
\end{array}$ \\
\hline SPTBN4 & R527W & NA & NR & Del. & $\begin{array}{l}\text { GScore: } 101 \\
\text { Pp: } 0.99\end{array}$ & VUS & $\begin{array}{l}\text { Congenital myopathy } \\
\text { with neuropathy, AR }\end{array}$ \\
\hline HSPG2 & R2196W & rs566319401 & 0.003 & Del. & $\begin{array}{l}\text { GScore: } 101 \\
\text { Pp: } 0.77 \\
\end{array}$ & VUS & $\begin{array}{c}\text { Schwartz-Jampel } \\
\text { syndrome, AR }\end{array}$ \\
\hline$D S G$ & $\mathrm{~L} 171 \mathrm{~F}$ & rs199926617 & 0.002 & Del. & $\begin{array}{l}\text { GScore: } 22 \\
\text { Pp: } 0.50\end{array}$ & VUS & $\begin{array}{l}\text { Arrhythmogenic } \\
\text { Right Ventricular } \\
\text { Dysplasia, AD/AR }\end{array}$ \\
\hline
\end{tabular}

NA or NR - Not available or not reported

${ }^{b}$ Del. - Deleterious; LoF - Loss of function; GScore: Grantham Score scores substitutions according to the degree of the physico- chemical difference between the original and the new amino acid; Pp: The probability of the prediction, a value close to 1 indicates a high probability of pathogenicity.

${ }^{\mathrm{c}} \mathrm{AD}$ - Autosomal Dominant; AR- Autosomal Recessive.

another member of voltage-gated sodium channels, specifically SCN5A, have been associated with cardiomyopathies $[19,20]$. These results suggest that p. R838Q in SCN11A is likely pathogenic variant. Paralogue analysis was extended to all known disease-associated variants in SCN11A as well as variant locations in Nav1.9 (Figure 1, Table S).

\section{Discussion}

Exertional rhabdomyolysis often occurs in military service members engaged in strenuous physical activity. It has many potential causes that can be divided into acquired and inherited $[5,21]$. The service member in this study, who was SCT positive, presented as having had a single episode of exertional rhabdomyolysis with severe muscle pain, peak CK of 18,867U/L, and hematuria after exercise. Muscle enzymes and histopathology were essentially normal, thus ruling out metabolic and mitochondrial myopathies commonly associated with exertional rhabdomyolysis [5, 21]. This result was consistent with genetic results, which were negative for any predicted or known pathogenic variants associated with rhabdomyolysis. Detailed analysis of the clinical history revealed use of several prescription medications including amlodipine, simvastatin, and sertraline. Statin use is known to be associated with mild muscle complaints that in combination with amlodipine significantly increase the risk for acute rhabdomyolysis [6]. As a result, the daily dose of simvastatin is restricted to $\leq 20 \mathrm{mg}$ when taking amlodipine. Patient was taking simvastatin within the restricted dosage, but he was also taking sertraline $(100 \mathrm{mg})$, antidepressant selective serotonin reuptake inhibitor [22]. The daily dose of sertraline reported in association with exertional rhabdomyolysis ranges from 50-150 mg [22-24]. Rhabdomyolysis has also been precipitated by concomitant use of sertraline and other drugs, including amlodipine and simvastatin [22, 24]. In most reported cases, strenuous exercise appears to be a common thread in sertraline-induced rhabdomyolysis [22, 23]. These results suggest that sertraline and/or drug-to-drug interactions, together with the stress of exercise, may have contributed to the development of exertional rhabdomyolysis in this case.

The service member's history of recurrent hematuria and transient proteinuria triggered by exercise is significant. WES analysis revealed that the warfighter was a carrier of p. V260E, a known pathogenic variant in NPHS2 encoding Podocin, a glomerular podocyte protein that serves a key role in the regulation of glomerular permeability [14, 25]. Podocin abnormality typically results in dysfunction of the glomerular filtration barrier and development of proteinuria. Biallelic mutations in NPHS2 cause a nephropathy known as steroidresistant nephrotic syndrome (SRNS), which is characterized by massive proteinuria, hypoalbuminemia, and edema [14]. The inheritance of pathogenic NPHS2 p. V260E mutation likely contributed to the patient's recurrent hematuria and proteinuria. Given SCT status and history of recurrent exercise-induced hematuria and proteinuria, it is likely that coinheritance of p. V260E in NPHS2 and HbAS likely have cumulative effects and compromised kidney function upon exertion.

Another key finding was the identification of the SCN11A p. R838Q variant. Patient had reported severe and burning pain in his calves triggered by exercise. His pain, localized 


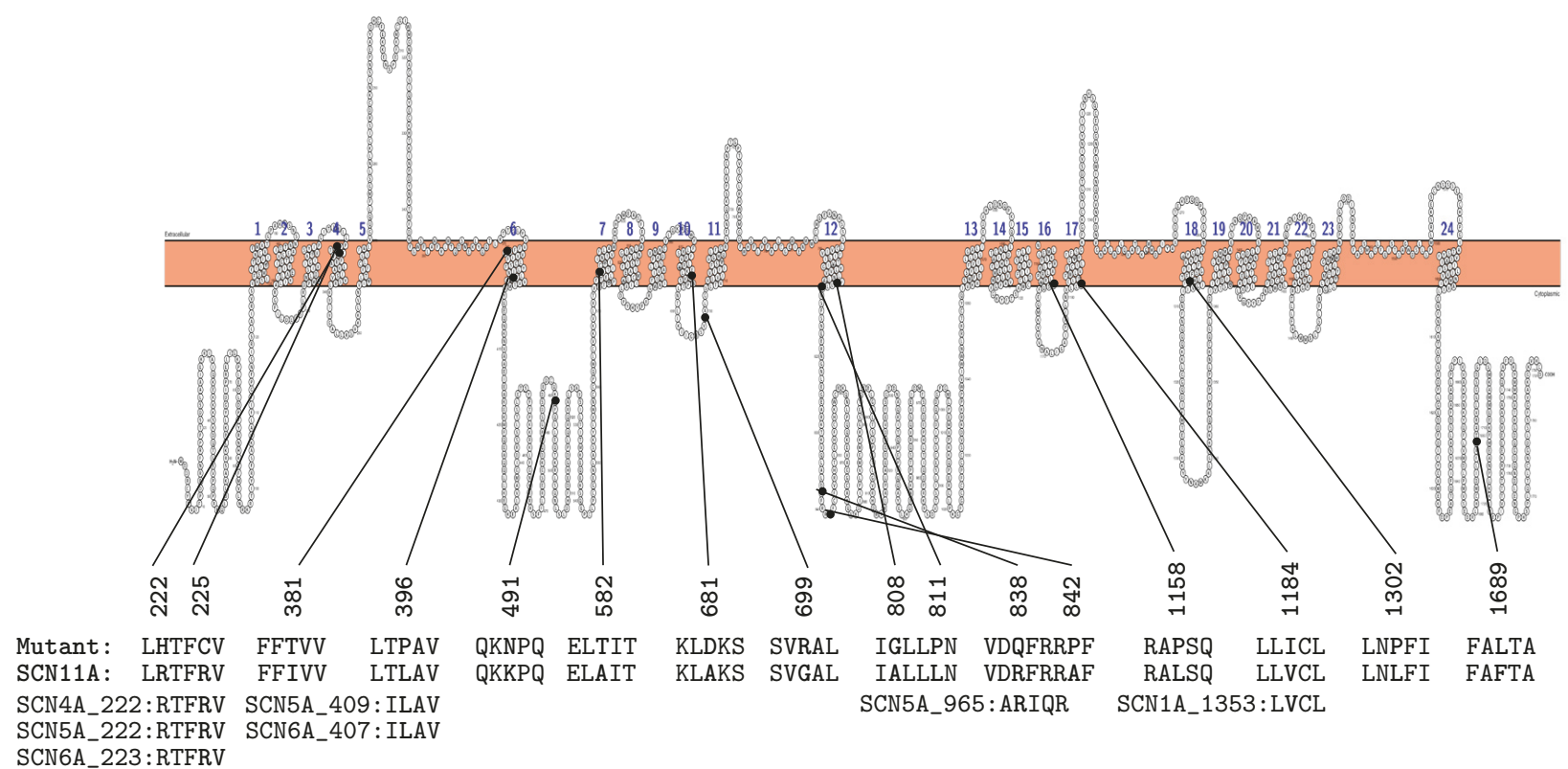

FIGURE 1: Locations and paralogue analysis of SCN11A variants associated with pain diseases in the Nav1.9 channel. The Nav1.9 channel, like other sodium channels, is composed of 24 transmembrane segments organized into four domains. Mutated residues are in bold, black dots represent locations of mutated residues in the channel. Amino acid residue number is given above mutated residue. Paralogue sodium channels along with mutated residues and their numbers are given below equivalent residues in SCN11A.

predominantly in the distal lower extremity, was distinct from the abdominal and chest pain due to splenic infarction and pulmonary embolism in SCT individuals $[1,3]$. In addition, his leg pain was unique from exertional leg pain in SCT individuals we have previously seen clinically in its atypical presentation in lasting days in duration. Pathogenic variants in SCN11A are associated with a range of autosomal dominant syndromic pain diseases such as episodic pain, painful neuropathy, and insensitivity to pain $[12,13,26]$. The p. R838Q variant located in close proximity to three other SCN11A pathogenic variants, including A808G and A842P, reported in association with episodic pain and painful neuropathy respectively $[12,13]$. The $\mathrm{p}$. R838Q variant like other pathogenic variants, p. R225C, p. I381T, p. L396P and p. V1184A, in SCN11A was annotated with paralogue mutations in functionally similar members of the voltage-gated sodium channel family (Figure 1). There is also striking resemblance between episodic pain described in SCN11A families and the pain phenotype manifested in the reported case. SCN11Aassociated episodic pain is characterized by severe burning pain predominantly in the lower extremities [12, 13]. Pain occurs episodically, typically once every 2-5 days and can be triggered or exacerbated by fatigue or heavy exercise. We suggest that $\mathrm{p}$. R838Q variant, similarly with other SCN11A pathogenic variants, may cause neuronal hyperexcitability and lead to the service member's episodes of severe burning calf pain. Further studies of the functional effect of this variant in episodic pain are warranted.

Development of exertional rhabdomyolysis and hematuria in SCT individuals is thought to occur due to polymerization of $\mathrm{HbAS}$ and red blood cell sickling $[1,3]$. However, whether HbAS-related red blood sickling is the cause of these complications remains controversial. Exercise studies have demonstrated no difference in muscle microvasculature, blood rheology, and inflammatory factors between individuals with and without SCT [27]. Epidemiological studies have shown that exertional rhabdomyolysis and hematuria occur in $1.2 \%$ and $2.5 \%$ of African Americans with SCT respectively [28,29]. The fact that not all SCT carriers develop exertional rhabdomyolysis and hematuria indicates a variable degree of risks and expressions of these complications among SCT individuals. Importantly, this highlights a role of other environmental and genetic factors as presented in the case under the study. The service member was taking a combination of drugs known to be associated with an increased risk for exertional rhabdomyolysis suggesting that drug-to-drug interactions, together with the stress of exercise, may have contributed to the development of exertional rhabdomyolysis in this case. Coinheritance of HbAS and NPHS2 p.V260E, rather than sickling alone, may be the underlying cause of recurrent hematuria and transient proteinuria. The likely pathogenic variant p. R838Q in SCN11A may explain, in part, the patient's episodic burning pain affecting his calf muscles.

In conclusion, we identified a SCT positive case who presented with exertional rhabdomyolysis, recurrent hematuria, and episodic burning pain in the lower extremities. Clinical and genetic studies revealed the multifactorial nature of his clinical presentations with the role of both acquired and inherited factors in the observed phenotypes. This case underscores the importance of comprehensive clinical and genetic evaluations to identify underlying causes of health complications reported in SCT individuals. 


\section{Conflicts of Interest}

The information presented represents the opinions of the authors and not those of the Department of Defense or the Uniformed Services University. We have no financial interests or relationships to disclose.

\section{Acknowledgments}

The authors are grateful to the Collaborative Health Initiative Research Program, USU, for exome sequencing and the Biomedical Instrumental Center, USU, for Sanger sequencing and oligo synthesis. We thank Dr. Kristin Heitman for her critical comments and Dr. Dale Szpisjak and Ms. Maria Voelkel for their support and assistance. The study was supported by the National Heart, Lung and Blood Institute grant agreement HU0001-14-1-0060 to Dr. P. Deuster, and by USU, Intramural Research Program to Dr. F. O'Connor.

\section{Supplementary Materials}

Supplementary materials include muscle histopathology images (Fig. S1), integrated view of exome sequencing results (Fig. S2), amino acid alignments of regions with substituted residues in the voltage-gated sodium channels (Fig. S3), and genetic variant analysis and citations. (Supplementary Materials)

\section{References}

[1] N. S. Key and V. K. Derebail, "Sickle-cell trait: novel clinical significance," American Society of Hematology, vol. 2010, no. 1, pp. 418-422, 2010.

[2] J. Ojodu et al., "Incidence of sickle cell trait-United States," Morbidity and Mortality Weekly Report, vol. 63, no. 49, pp. 1155$1158,2010$.

[3] R. P. Naik and V. K. Derebail, "The spectrum of sickle hemoglobin-related nephropathy: from sickle cell disease to sickle trait," Expert Review of Hematology, vol. 10, no. 12, pp. 1087-1094, 2017.

[4] F. G. O'connor, M. F. Bergeron, J. Cantrell et al., "ACSM and CHAMP Summit on Sickle Cell Trait," Medicine \& Science in Sports \& Exercise, vol. 44, no. 11, pp. 2045-2056, 2012.

[5] J. R. Nance and A. L. Mammen, "Diagnostic evaluation of rhabdomyolysis," Muscle \& Nerve, vol. 51, no. 6, pp. 793-810, 2015.

[6] D. Hilton-Jones, "Statin-related myopathies," Practical Neurology, vol. 18, no. 2, pp. 97-105, 2018.

[7] R. Peces, C. Peces, E. Cuesta-López et al., "Co-inheritance of autosomal dominant polycystic kidney disease and sickle-cell trait in African Americans," Nefrología, vol. 31, no. 2, pp. 162168, 2011.

[8] R. P. Naik et al., "Sickle cell trait and the risk of ESRD in blacks," Journal of the American Society of Nephrology, vol. 28, no. 7, pp. 2180-2187, 2017.

[9] K. Abdool, K. Ramcharan, A. J. Reyes, N. Lutchman, and A. Alexander, "Bilateral pulmonary embolism after a short-haul flight in a man with multiple risk factors including sickle cell trait," Case Reports in Emergency Medicine, vol. 2017, Article ID 4316928, 5 pages, 2017.
[10] M. Sinha, B. Raghuwanshi, N. Bag, and A. Barman, "Splenic infarction in two members of the family with sickle cell trait: A case report of rare complication," International Journal of Applied and Basic Medical Research, vol. 7, no. 4, p. 272, 2017.

[11] D. L. H. Bennett and C. G. Woods, "Painful and painless channelopathies," The Lancet Neurology, vol. 13, no. 6, pp. 587599, 2014.

[12] S. D. Dib-Hajj, J. A. Black, and S. G. Waxman, "Na v 1.9: A sodium channel linked to human pain," Nature Reviews Neuroscience, vol. 16, no. 9, pp. 511-519, 2015.

[13] X. Zhang, J. Wen, W. Yang et al., "Gain-of-Function Mutations in SCN11A Cause Familial Episodic Pain," American Journal of Human Genetics, vol. 93, no. 5, pp. 957-966, 2013.

[14] K. Bouchireb et al., "NPHS2 mutations in steroid-resistant nephrotic syndrome: a mutation update and the associated phenotypic spectrum," Human Mutation, vol. 35, no. 2, pp. 178186, 2014.

[15] N. Sambuughin, E. Zvaritch, N. Kraeva et al., "Exome analysis identifies Brody myopathy in a family diagnosed with malignant hyperthermia susceptibility," Molecular Genetics \& Genomic Medicine, vol. 2, no. 6, pp. 472-483, 2014.

[16] S. Richards, N. Aziz, and S. Bale, "Standards and guidelines for the interpretation of sequence variants: a joint consensus recommendation of the American College of Medical Genetics and Genomics and the Association for Molecular Pathology," Genetics in Medicine, vol. 17, no. 5, pp. 405-423, 2015.

[17] R. Walsh, N. S. Peters, S. A. Cook, and J. S. Ware, "Paralogue annotation identifies novel pathogenic variants in patients with brugada syndrome and catecholaminergic polymorphic ventricular tachycardia," Journal of Medical Genetics, vol. 51, no. 1, pp. 35-44, 2014.

[18] U. Omasits, C. H. Ahrens, S. Müller, and B. Wollscheid, "Protter: Interactive protein feature visualization and integration with experimental proteomic data," Bioinformatics, vol. 30, no. 6, pp. 884-886, 2014.

[19] C.-H. Hsueh, W.-P. Chen, J.-L. Lin et al., "Distinct functional defect of three novel Brugada syndrome related cardiac sodium channel mutations," Journal of Biomedical Science, vol. 16, no. 1, article no. 23, 2009.

[20] J. D. Kapplinger, D. J. Tester, M. Alders et al., "An international compendium of mutations in the SCN5A-encoded cardiac sodium channel in patients referred for Brugada syndrome genetic testing," Heart Rhythm, vol. 7, no. 1, pp. 33-46, 2010.

[21] R. S. Scalco, M. Snoeck, R. Quinlivan et al., "Exertional rhabdomyolysis: physiological response or manifestation of an underlying myopathy?" BMJ Open Sport \& Exercise Medicine, vol. 2, no. 1, p. e000151, 2016.

[22] M. Snyder and T. Kish, "Sertraline-induced rhabdomyolysis: A case report and literature review," American Journal of Therapeutics, vol. 23, no. 2, pp. e561-e565, 2016.

[23] M. Labotz, T. K. Wolff, K. T. Nakasone, I. F. Kimura, R. K. Hetzler, and A. W. Nichols, "Selective serotonin reuptake inhibitors and rhabdomyolysis after eccentric exercise," Medicine \& Science in Sports \& Exercise, vol. 38, no. 9, pp. 15391542, 2006.

[24] P. Gareri, C. Segura-Garcia, P. De Fazio, S. De Fazio, and G. De Sarro, "Sertraline-induced rhabdomyolysis in an elderly patient with dementia and comorbidities," Annals of Pharmacotherapy, vol. 43, no. 7-8, pp. 1354-1359, 2009.

[25] S. Weber, O. Gribouval, E. L. Esquivel et al., "NPHS2 mutation analysis shows genetic heterogeneity of steroid-resistant 
nephrotic syndrome and low post-transplant recurrence," Kidney International, vol. 66, no. 2, pp. 571-579, 2004.

[26] E. Leipold, L. Liebmann, G. C. Korenke et al., "A de novo gainof-function mutation in SCN11A causes loss of pain perception," Nature Genetics, vol. 45, no. 11, pp. 1399-1407, 2013.

[27] J. Tripette et al., "Exercise-related complications in sickle cell trait," Clin Hemorheol Microcirc, vol. 55, no. 1, pp. 29-37, 2013.

[28] D. A. Nelson, P. A. Deuster, and L. M. Kurina, "Sickle cell trait and rhabdomyolysis among U.S. Army Soldiers: The authors reply," The New England Journal of Medicine, vol. 375, no. 17, p. 1696, 2016.

[29] P. Heller, W. R. Best, R. B. Nelson, and J. Becktel, "Clinical Implications of Sickle-Cell Trait and Glucose-6-Phosphate Dehydrogenase Deficiency in Hospitalized Black Male Patients," The New England Journal of Medicine, vol. 300, no. 18, pp. 1001-1005, 1979. 


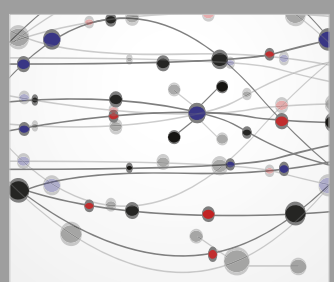

The Scientific World Journal
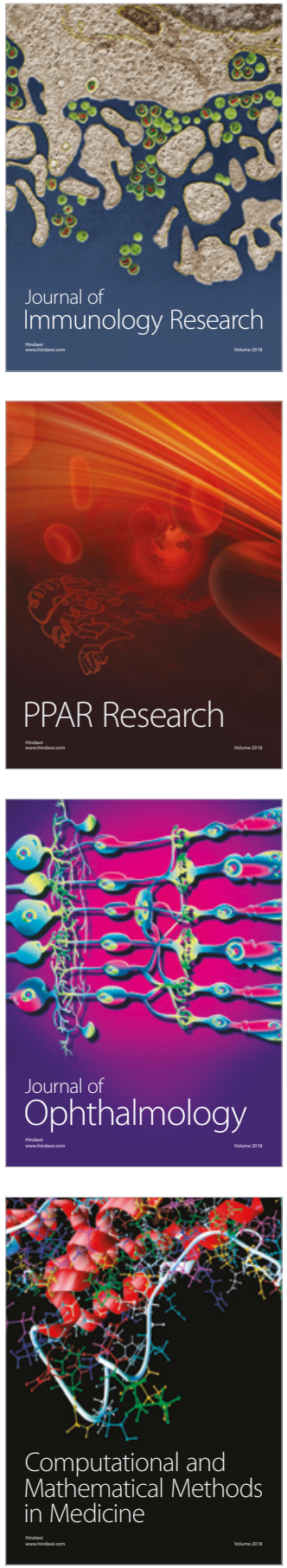

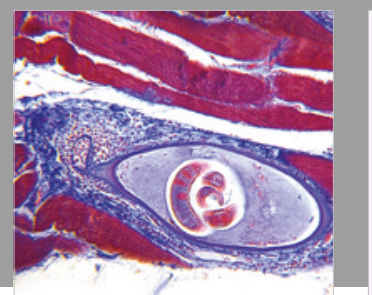

Gastroenterology Research and Practice

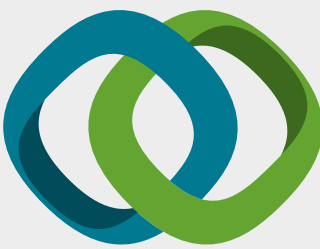

\section{Hindawi}

Submit your manuscripts at

www.hindawi.com
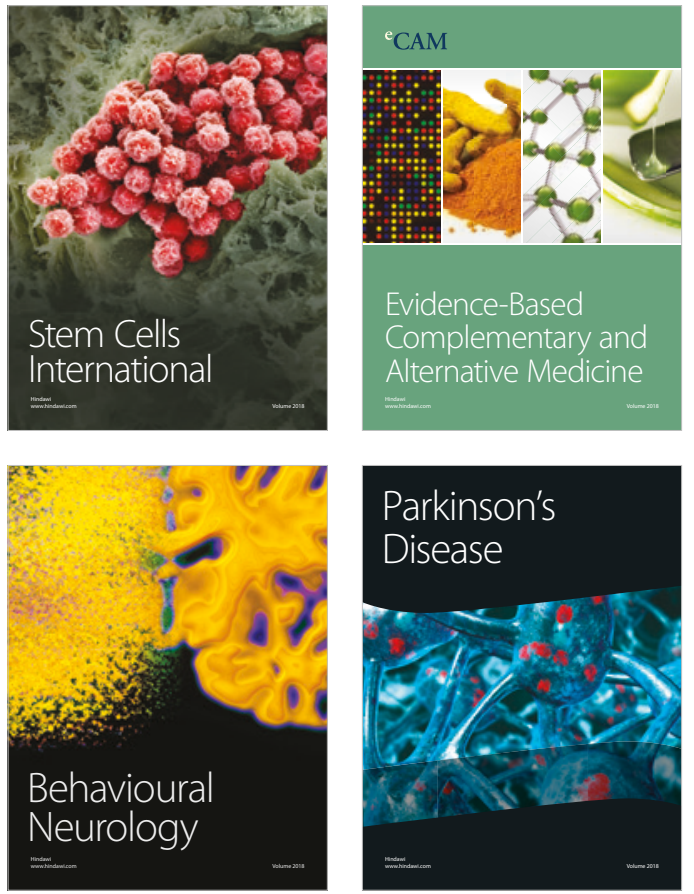

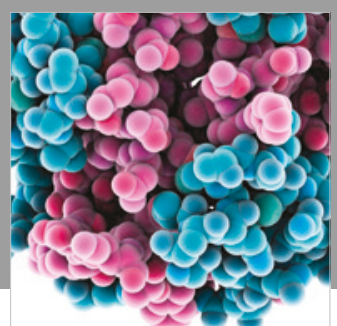

ournal of

Diabetes Research

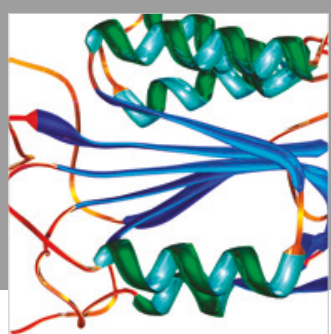

Disease Markers
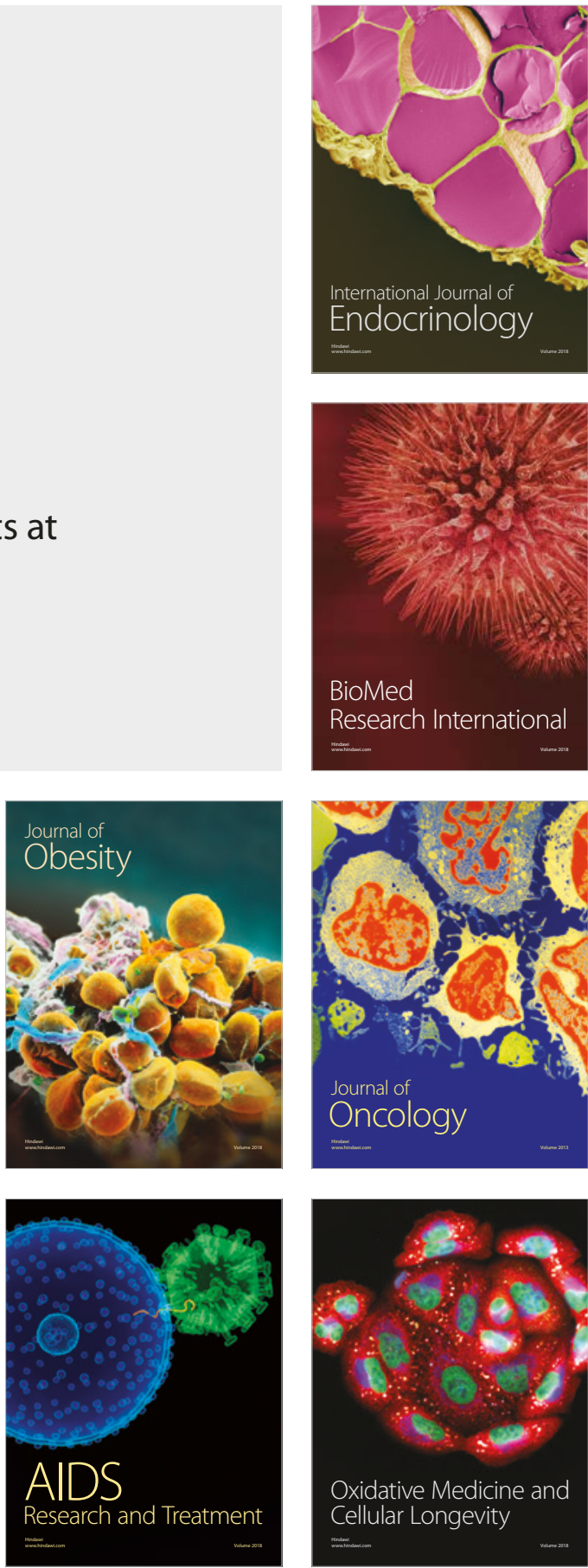